\title{
Risk factors of 90-day rehospitalization following discharge of pediatric patients hospitalized with mycoplasma Pneumoniae pneumonia
}

\author{
Le Wang ${ }^{1 \dagger}$, Zhishan Feng ${ }^{2 \dagger}$, Jinfeng Shuai ${ }^{3}$, Jianhua Liu ${ }^{3^{*}}$ and Guixia $\mathrm{Li}^{1^{*}}$ (D)
}

\begin{abstract}
Background: Among pediatric patients hospitalized for Mycoplasma pneumoniae pneumonia (MPP), the risk factors for 90-day readmission after discharge is undefined.

Methods: We conducted a retrospective observational study of patients $<14$ years of age who were discharged with a diagnosis of MPP between January 2016 and February 2017. We collected clinical, laboratory and radiographic variables at the time of initial admission. We assessed pneumonia-related readmission within 90-day after discharge. Risk factors independently associated with rehospitalization were identified using multiple logistic regression models.

Results: Of the 424 MPP hospitalizations, 48 (11.3\%) were readmitted within 90 days and were mainly diagnosed with pneumonia. Patients with younger age or coinfection with influenza A were more likely to be readmitted. In addition, compared with children without readmission, the readmission ones showed different clinical and laboratory characteristics at the index hospital admission. Multiple logistic regression analysis identified age (OR $0.815,95 \% \mathrm{Cl} 0.706-0.940)$ and body temperature (OR $0.659,95 \% \mathrm{Cl} 0.518-0.839)$ were significantly associated with lower risk of 90-day readmission. Coinfection with influenza was independently associated with a greater likelihood of 90-day readmission (OR 4.746, 95\%Cl 1.191-18.913).

Conclusions: Readmission after MPP are common and is related to patients' age, body temperature and influenza A coinfection during initial hospital stay, indicating potential targets could be noticed to reduce the rehospitalization after pediatric MPP.
\end{abstract}

Keywords: MPP, Rehospitalization, Children

\section{Introduction}

Readmission of patients initially hospitalized for community acquired pneumonia (CAP) is relatively common [1-3]. Both of preventable and non-preventable risk factors have been explored, but the main participants in these studies were the elderly and patients with multiple comorbidities, not children [4-7].

\footnotetext{
* Correspondence: hbetliujh@126.com; hbetlgx@126.com

${ }^{\dagger}$ Le Wang and Zhishan Feng contributed equally to this work.

${ }^{3}$ No.2 Department of Respiratory, Children's Hospital of Hebei Province, 133

Jianhua South Street, Shijiazhuang 050031, Hebei Province, China

${ }^{1}$ Institute of Pediatric Research, Children's Hospital of Hebei Province, 133

Jianhua South Street, Shijiazhuang 050031, Hebei Province, China

Full list of author information is available at the end of the article
}

The previous three studies described 14 days [8], 30 days [9] readmission rates (range of 1.5-8\%) for children with pneumonia or lower respiratory infections (LRIs) [10]. In these studies, one of the consistent identified risk factors was chronic medical conditions. But in fact, a large number of children who are rehospitalized are caused by acute diseases [11]. If patients with chronic conditions are excluded, the difficulty is to detect readmission risk factors associated with the current acute infection. In addition, children with LRIs, a relatively broad diagnosis, or pneumonia with an underlying chronic illness may bring compounding factors conferring susceptibility for readmission. A promising approach to resolve this problem is to

(c) The Author(s). 2019 Open Access This article is distributed under the terms of the Creative Commons Attribution 4.0 International License (http://creativecommons.org/licenses/by/4.0/), which permits unrestricted use, distribution, and reproduction in any medium, provided you give appropriate credit to the original author(s) and the source, provide a link to the Creative Commons license, and indicate if changes were made. The Creative Commons Public Domain Dedication waiver (http://creativecommons.org/publicdomain/zero/1.0/) applies to the data made available in this article, unless otherwise stated. 
narrow down study sample according to the pathogenic or clinical features, such as Mycoplasma pneumoniae pneumonia (MPP), which accounts for up to $40 \%$ pediatric CAP [12], and its diagnosis is based on etiology and clinical evidence, thereby elevating the power to detect readmission risk factors associated with the current acute infections. We hypothesized that MPP children may have different characteristic during the first hospital stay between patients with and without readmission. This study was therefore conducted to enroll pediatric MPP patients without other underlying chronic diseases.

Our aims were to (1) describe the incidence and type of readmission after MPP discharge, (2) investigate the differences between patients with and without readmission at the initial hospital stay, (3) examine the risk factors for 90-day pneumonia-related rehospitalization.

\section{Material and methods Study design}

This retrospective, observational study was conducted at Children's Hospital of Hebei Province, a 1200-bed teaching hospital in Hebei Province (northern China) that serves a population of 70,000,000 inhabitants, including $18.5 \%$ children. Patients with a discharge diagnosis of MPP were evaluated. The project was approved by the ethics review board of the hospital. Because data in this report were collected from inpatient electronic medical records, there was no need to collect new specimens or the corresponding written informed consent.

\section{Study sample}

Children $\leq 14$ years of age who were admitted to Children's Hospital of Hebei Province with a diagnosis of MPP from January 2016, to February 2017, were consecutively enrolled into the study. The diagnosis of MPP needs to meet the following first 2 points plus either third or fourth point [13]: 1) a new infiltrate on a chest radiograph; 2) fever, cough and abnormal lung auscultation; 3) positive serology laboratory results specific MP antibody titer $\geq 1: 160$ detected by a micro-particle agglutination test [14]; 4) positive PCR laboratory results, MP-DNA positive detected in sputum, nasopharyngeal aspirate or bronchoalveolar lavage fluid (BALF) by PCR [15]. Patients were excluded from the study if they were known to be chronically immunosuppressed, or with chronic cardiopulmonary conditions or had been hospitalized for the previous 14 days. If a patient had more than one episode of pneumonia during the index hospitalization, only the first one was included in the analysis.

\section{Data and specimen collection}

The following patient characteristics were evaluated: age, sex, signs and symptom before admission (wheezing, cough and diarrhea). The laboratory data and radiological findings were also measured and retrospectively investigated from inpatient electronic medical records system. Clinical symptoms included wheezing, cough and diarrhea. Ill day and febrile day before admission, hospital days, febrile day and readmission rate were also recorded. The time frame of readmission was set as 90day from original discharge. Body temperature was examined at the beginning of admission and every $8 \mathrm{~h}$ thereafter. A febrile day was defined as the body temperature exceeded $38.0^{\circ} \mathrm{C}$ at least once [16].

Patients were asked to cough, and the expectorated sputum was collected. If the child is too young to cough, a sterile negative pressure suction catheter is applied to obtain the oropharyngeal suction (OPS). The storage, transportation and nucleic acid extraction procedure were reported elsewhere [17]. The paired serum samples were taken at the presentation of pneumonia and at least 7 days after the first collection of serum. The serum was obtained from $2 \mathrm{~mL}$ whole blood by the separation gel tube.

\section{Detection of MP-DNA and MP-antibody}

The GeXP assay (GenomeLab GeXP Genetic Analysis System) was performed on all specimens for the 13 type/ subtypes of common respiratory pathogens including $M$. pneumoniae. The multiplex-PCR was performed as previously described elsewhere [18]. The bacteria infection was examined by standard culture methods from sputum specimens [19]. The determination of MP-specific antibody was performed using a commercially available micro-particle agglutination test Serodia-MycoII kit (Fujirebio, Tokyo, Japan) [14]. Diagnosis criteria were defined as $\geq 4$-found rising for paired sera or single serum of titer $\geq 1: 160$ [15].

\section{Statistical analysis}

The chi-squared test was used to compare categorical variable in subgroups. And for those continuous variables with normal and non-normal distributions, mean or median values were compared using the $t$ test or Mann-Whitney U test. SPSS 19.0 statistics package (SPSS Inc., Chicago, USA) software was used for all statistical analysis. $p<0.05$ was considered statistically significant.

\section{Results}

Patients enrollment and readmission diagnoses

During the study period, 432 inpatients met our study eligibility criteria for MPP: 8 patients were excluded due to the missing sex, age or diagnostic data, and 368 patients were not readmitted to hospital within 90 days (Fig. 1). Of the 56 patients who were readmitted in 90 days, 8 were diagnosed with pneumonia-unrelated 


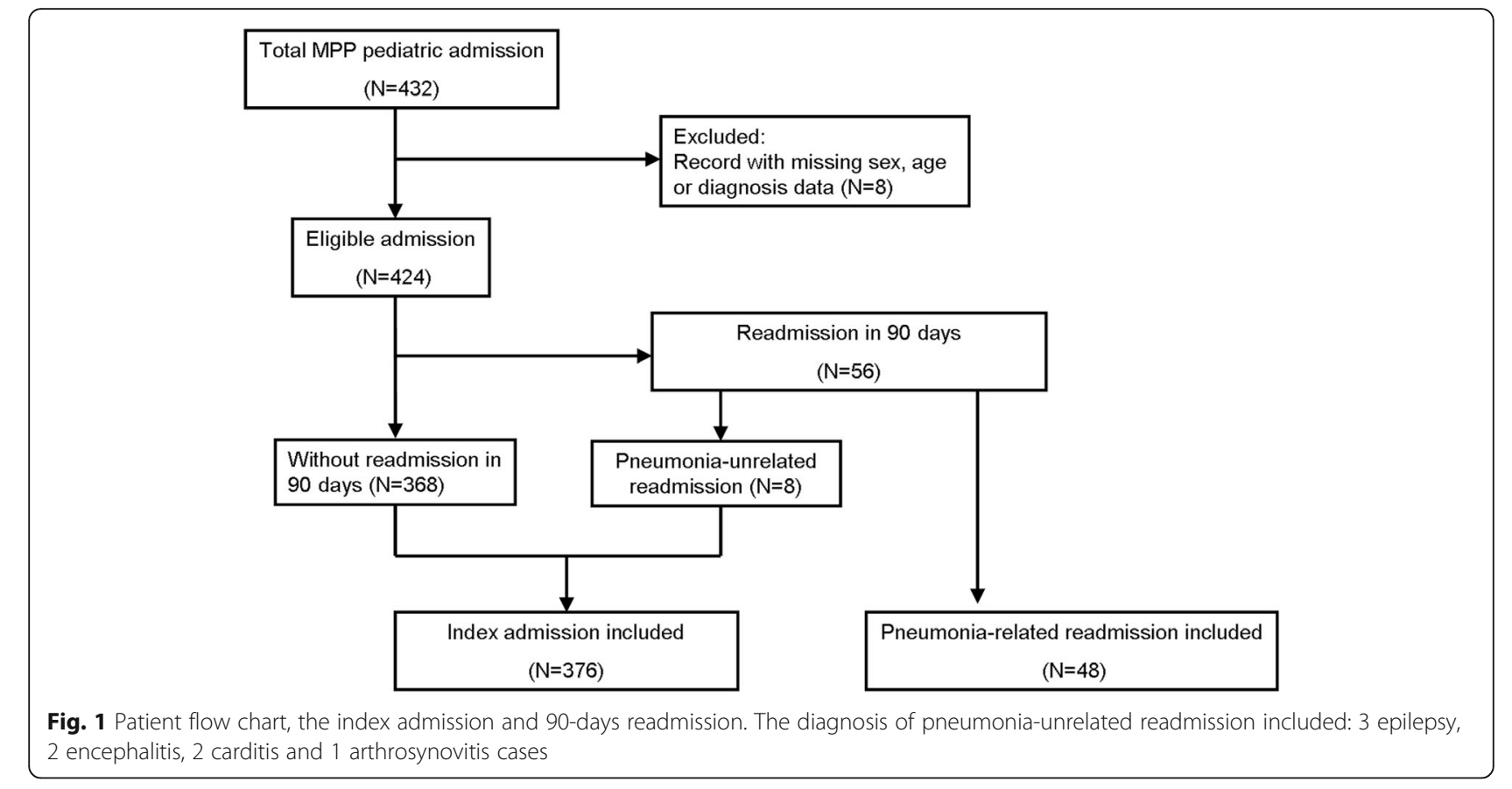

diseases including epilepsy, encephalitis, carditis and arthritis. Therefore, 48 (11.3\%) children were readmitted due to pneumonia-related diseases in 90 days of initial MPP discharge. Of the 48 re-admitted cases, 17 (35.4\%) were reinfected with MP, 23 (47.9\%) were negative for MP, and 8 (16.7\%) did not receive pathogen detection test. The most common cause of readmission was pneumonia (47.9\%), followed by bronchial pneumonia (45.8\%) bronchitis (4.2\%) and one (2.1\%) refractory mycoplasma pneumoniae pneumonia (RMPP) (Table 1). Among the enrolled 424 patients, there were 23 cases were detected to be positive by PCR alone, 129 were serology alone (with 6 cases as seroconversion and 123 cases as single high titer) and 272 were positive by both PCR and serology assays.

\section{Characteristics of children}

Demographic characteristics included age (median age 4.5, range in 0.1-13) and gender (238 boys and 186 girls). As shown in Table 2, patients who were readmitted in 90 days were significantly younger than those without readmission (median age: 2 v.s. 4.7 years, $p<0.001$ ) and were more likely

Table 1 The diagnosis of 90-days pneumonia-related readmission

\begin{tabular}{lll}
\hline Readmission diagnosis & No. & $\%$ \\
\hline Pneumonia & 23 & 47.9 \\
Bronchopneumonia & 22 & 45.8 \\
Bronchitis & 2 & 4.2 \\
Refractory Mycoplasma pneumoniae pneumonia & 1 & 2.1 \\
\hline
\end{tabular}

to show wheezing symptoms $(p<0.001)$, as well as a lower body temperature on admission $(p<0.001)$, shorter febrile days during hospital stay $(p<0.001)$. Similarly, more patients with readmission showed normal $(p=0.009)$ or light diffuse shadowing $(p=0.030)$ radiological findings. CRP, $\mathrm{LDH}, \mathrm{HBDH}$ and neutrophil percentage levels were lower, but the percentage of lymphocyte was significantly greater (all $p<0.05$ ) (Table 3).

\section{Coinfection pathogens}

Coinfection was observed in 189 (44.6\%) cases, and children infected with influenza A were more likely to be admitted again $(p<0.001$, Table 4$)$. Coinfection rates of other pathogens (rhinovirus, parainfluenza, influenza B, respiratory syncytial virus, adenovirus, coronavirus, human metapneumovirus, S. pneumoniae and human bocavirus) did not vary significantly between patients with and without rehospitalization.

\section{Multiple logistic regression analysis}

Multiple logistic regression analysis identified age (OR 0.815 , 95\% CI $0.706-0.940$ ) and body temperature (OR $0.659,95 \% \mathrm{CI} 0.518-0.839$ ) during initial hospital stay were significantly associated with lower risk of 90-day readmission. Coinfection with influenza A at index admission was independently associated with a greater likelihood of 90day readmission (OR 4.746, 95\%CI 1.191-18.913) (Table 5).

\section{Discussion}

Generally, readmissions after pneumonia are common. In terms of safety and cost, it is important to assess the 
Table 2 Patient characteristic on index visit are different between children with and without readmission

\begin{tabular}{|c|c|c|c|c|}
\hline & & $\begin{array}{l}\text { With readmission } \\
n=48\end{array}$ & $\begin{array}{l}\text { Without readmission } \\
n=376\end{array}$ & $P$ \\
\hline \multirow[t]{2}{*}{ Sociodemographic } & Sex (Male) & $31(64.6 \%)$ & $211(56.1 \%)$ & 0.268 \\
\hline & Age (Year) & $2(1-4.6)$ & $4.7(2.5-7)$ & $<0.001$ \\
\hline \multirow[t]{5}{*}{ Before and after admission } & III day before admission & $10(5.5-15)$ & $8(6-14)$ & 0.449 \\
\hline & Febrile day before admission & $3(0-8.5)$ & $6(3-10)$ & 0.002 \\
\hline & Body Temperature on admission & $38.5(36-39.4)$ & $39.3(38.6-39.8)$ & $<0.001$ \\
\hline & Hospital day & $9(7-15)$ & $10(7-14)$ & 0.819 \\
\hline & Febrile day after admission & $1(0-3)$ & $2(0-4)$ & $<0.001$ \\
\hline \multirow[t]{3}{*}{ Clinical manifestation at presentation } & Wheezing day & $5(3-8)$ & $3(2-10)$ & $<0.001$ \\
\hline & Cough day & $9(4.5-15)$ & $7(5-12)$ & 0.532 \\
\hline & Diarrhea day & $6(5-7)$ & $2.5(2-3)$ & 0.397 \\
\hline \multirow[t]{4}{*}{ Radiographic finding } & Normal & $11(22.9 \%)$ & $38(10.1 \%)$ & $0.001^{\mathrm{a}}$ \\
\hline & Light diffuse shadowing & $15(31.2 \%)$ & $68(18.1 \%)$ & \\
\hline & Consolidation & $16(33.3 \%)$ & 169 (44.9\%) & \\
\hline & Pleural effusion & $5(10.4 \%)$ & $75(19.9 \%)$ & \\
\hline \multirow[t]{2}{*}{ Severity on admission } & ICU admission & $7(14.6)$ & $26(6.9 \%)$ & 0.114 \\
\hline & Mechanical ventilation & $3(6.3 \%)$ & $13(3.5 \%)$ & 0.580 \\
\hline
\end{tabular}

Data were shown in median (IQR) or N (\%). Comparative analysis was performed using the Chi-Square or Mann-Whitney U test, as appropriate

${ }^{a}$ Separately, $p$ values are 0.009 for Normal group, 0.030 for Light diffuse shadowing group, 1.127 for Consolidation group, 0.112 for Pleural effusion group

relationship between initial hospital stay and readmission outcomes $[9,20]$. Although the investigation of risk factors is challenging, significant progress has been made on the elderly, that the readmission was found to be largely depends on the comorbidities and factors external to the patient $[1,2,4,21]$. This has also been observed in children, and one of the identified risk factors is chronic medical conditions such as underlying pulmonary or cardiovascular disease [9-11]. To date, research on potential risk factors for readmission has hardly focused on current acute infections or specific pathogens. Because pneumonia is a complex heterogenous disease that can be caused by a variety of pathogens, 1) studying pneumonia

Table 3 Different laboratory data on index hospitalization between patients with and without readmission

\begin{tabular}{llll}
\hline Laboratory data & With readmission & Without readmission & $P$ \\
& $n=48$ & $n=376$ & \\
\hline WBC (10^9/L) & $8.6(7.3,11.3)$ & $9.9(7.6,13.2)$ & 0.192 \\
Neutrophil (\%) & $49.2 \pm 17.5$ & $55.6 \pm 16.3$ & 0.024 \\
Lymphocyte (\%) & $40.4 \pm 16.0$ & $31.9(21.3,43.6)$ & 0.008 \\
Monocyte (\%) & $7.1 \pm 2.6$ & $6.6(5.4-8.2)$ & 0.637 \\
CRP (mg/L) & $4.2(1.0,11.4)$ & $10.4(1.4-30.0)$ & 0.025 \\
LDH (IU/L) & $261.5(228.7,299.2)$ & $290.0(244.7-373.0)$ & 0.009 \\
HBDH (IU/L) & $211.5(189.0,236.2)$ & $237.5(201.7-296.0)$ & 0.005 \\
\hline WBC Whis
\end{tabular}

WBC White blood cell, $L D H$ Lactatedehydrogenase, $H B D H$ Hydroxybutyrate dehydrogenase, CRP C-reactive protein

Data were shown in mean \pm SD or median (IQR)

Comparative analysis was performed using Chi-Square or Mann-Whitney $\mathrm{U}$ test patients with the same infectious pathogen can reduce heterogeneity, 2) exclusion of patients with underlying diseases can improve the ability to detect readmission risk factors associated with the current acute infections. Therefore, we investigated the rate of 90-day pneumoniarelated readmission in hospitalized children with MPP who had no basal or chronic disease. After comparing the clinical information of the first hospitalization between patients who were readmitted and not readmitted to the hospital, we obtained the following findings: 1) 48 (11.3\%) children were readmitted within 90 days of the first MPP

Table 4 The coinfection pathogens in patients with 90-days readmission

\begin{tabular}{llll}
\hline Pathogen & No. $^{\text {a }}$ & Readmission cases & $P$ \\
\hline Rhinovirus & 80 & $12(15.0 \%)$ & 0.249 \\
Parainfluenza & 33 & $5(15.2 \%)$ & 0.470 \\
Influenza B & 29 & $5(17.2 \%)$ & 0.297 \\
Respiratory syncytial virus & 17 & $1(5.9 \%)$ & 0.740 \\
Adenovirus & 15 & $2(13.3 \%)$ & 1.000 \\
Coronavirus & 13 & $1(7.7 \%)$ & 0.675 \\
Human metapneumovirus & 13 & $1(7.7 \%)$ & 1.000 \\
Influenza A & 11 & $6(54.5 \%)$ & $<0.001$ \\
S. pneumoniae & 10 & $1(10.0 \%)$ & 0.894 \\
Human Bocavirus & 6 & $0(0.0 \%)$ & 0.816 \\
\hline
\end{tabular}

aThere are mixed infections with more than one pathogen, the total cases is not equal to 189 . There are 155 cases of single pathogen coinfection, 30 cases of two pathogens, 4 cases of 3 pathogens 
Table 5 Stepwise logistic regression for the related factors associated with readmission

\begin{tabular}{lllll}
\hline Positive Variables & $P$-value & OR & \multicolumn{2}{l}{$95 \% \mathrm{Cl}$} \\
\cline { 4 - 5 } & & & Lower & Upper \\
\hline Age & 0.005 & 0.815 & 0.706 & 0.940 \\
$\begin{array}{l}\text { Influenza A } \\
\text { coinfection }\end{array}$ & 0.027 & 4.746 & 1.191 & 18.913 \\
$\begin{array}{l}\text { Body Temperature } \\
\text { on admission }\end{array}$ & 0.001 & 0.659 & 0.518 & 0.839 \\
\hline
\end{tabular}

discharge; 2) at the index hospital stay, readmission patients manifested different characteristics; 3) co-infection with influenza A increased the risk of 90-day readmission.

In this study, the readmission rate after MPP discharge was $11.6 \%$. Pneumonia and bronchial pneumonia were the majority diagnoses on readmission. Nakamura et al. identified that $5.5 \%$ cases readmitted after LRI hospitalization, and the most common readmission diagnose was LRI (48.2\%) [10]. Similar to the pattern observed by Neuman and colleagues, nearly half of the $8 \%$ of patients who were discharged from initial pneumonia hospitalization were also associated with pneumonia [9]. Previous work has shown that $30 \%$ pediatric readmissions are potentially preventable, especially the index admission and readmission are causally related [22]. This is one of the reasons we focus on pneumonia-related readmission. Meanwhile, we observed a trend in patients with relatively mild radiological symptoms and lower levels of acute inflammatory markers that are more likely to be rehospitalized. The cause of this phenomenon may be related to two points, including different host immunity and treatment strategies. First, clinical presentation depends on the host's immune response, rather than direct microbial destruction during the progression of M. pneumoniae infection [23]. Patient with a reduced immune system, such as younger ones who have had less time to develop immunity, may be characterized by mild clinical symptoms but with a prolonged recovery period. Second, pediatricians will adopt different treatment strategies for patients with severe or mild symptoms. Patients at risk of readmission may receive different medication times due to $M$. pneumoniae virulence or host immune response.

In this present study, we found that influenza coinfection increased the risk of readmission, which is consistent with previous investigations of children with complicated pneumonia. William et al. found that although there was a trend to increase mortality, patients with flu coinfection were less likely to readmitted in 2 weeks readmission, [24]. Brogan et al. observed that children who were infected with influenza during the initial hospital stay had a higher rate of readmission than children who were not infected with influenza [25]. Regarding the elderly, researches show that influenza vaccination is associated with a lower likelihood of readmission [5, 26, 27]. In view of these findings, influenza vaccination should be promoted not only in pediatric hospitals at CAP discharge, but also for all people, particularly in high risk groups including children under 5 years old, and those with asthma. In addition, we observed that younger children are liable to readmit, which is consistent with previous findings, demonstrating a higher rate of readmission for children under 1 year of age $[9,28]$. As explained by Gay JC et al., pneumonia in young patients usually has protracted and waning course, leading to structural lung damage or immune paresis and further pneumonia episodes [28]. Second, younger patients may be more prone to new infections due to higher exposure during nursery attendance and the previous lack of immunity to respiratory pathogens, which will be resulting in rehospitalization. Furthermore, Studies of children with asthma have found that the rate of readmission of children under 1 year of age in higher, further highlighting the need to improve inpatient decision-making for young patients.

To our knowledge, this is the first study to explore the factors of readmission for pediatric MPP patients. Further research in larger cohorts is needed to validate the data. Meanwhile, some questions remain to be answered: first, it has been reported that pneumonia attributed to potentially antibiotic-resistant bacteria is associated with an increased risk of readmission [21], we strongly felt that macrolide resistance has a role on the risk of readmission, but what is the role? Second, coinfection with influenza A will increase the risk of readmission, what is the underlying mechanism?

\section{Limitations}

This study has several limitations. First, the sample size may be small because only 48 patients were rehospitalized within 90 days. Second, if the child is readmitted to another institution, we may underestimate the rate of readmission. Third, in the absence of the information after first discharge, the interference factor related to age may be introduced into this research, as the rehospitalization may be caused by a new infection during nursery attendance. Fourth, other clinical information to document severity is not included in the study, such as oxygen requirement, antibiotic or corticoid duration. Fifth, a potential limitation would be that the serological assays have a high false positive detection rate and it is difficult to obtain the second serum. In our report, only 6 children provided paired sera, and the other 123 patients $(123 / 424,29 \%)$ had positive serological results with only a single high titer, but the PCR results were negative. Last, although there were significant differences in CRP, LDH levels or patient characteristics between rehospitalized and non-rehospitalized patients, these factors cannot be controlled and of low value in clinical practice. 


\section{Conclusions}

In conclusion, rehospitalization after MPP is relatively common and is related to patients' age and co-infected pathogens. Careful attention to clinical variables may reduce the frequency of rehospitalization of pediatric patients after discharge on MPP.

\section{Abbreviations}

CAP: Community acquired pneumonia; GeXP assay: GenomeLab Genetic Analysis System; MP or M. pneumoniae: Mycoplasma pneumoniae; RT-

PCR: Reverse transcription polymerase chain reaction

\section{Acknowledgements}

We sincerely thank all the participants for their support. We also thank Dr. Sukun Lu and Lijie Cao from the No.2 Department of Respiratory, Children's Hospital of Hebei Province, for their help to record the clinical information.

\section{Authors' contributions}

LW performed the statistical analysis and drafted the manuscript. ZF participated in the validation. $G L$ and $J$ participated in the design of the study. GL conceived of the study, and participated in its design and coordination, JS helped to draft the manuscript. All authors read and approved the final manuscript.

\section{Funding}

Not applicable.

\section{Availability of data and materials}

The datasets generated and/or analyzed during the current study are available in the (figshare) repository (https://figshare.com/articles/MP_ readmission/6838043). The data showed 424 cases with readmission and index hospitalization after MPP.

\section{Ethics approval and consent to participate}

The protocols used in this retrospective study was reviewed and approved by the institutional review board of Children's Hospital of Hebei Province. Because there was no need to collect new specimens and the clinical data was de-identified, so the consent was waived by IRB of Children's Hospital of Hebei Province. After obtaining the IRB' permission, we can review patient records and use these data, which were all de-identified.

\section{Consent for publication}

Not applicable.

\section{Competing interests}

All the authors declared that they have no competing interests.

\section{Author details}

${ }^{1}$ Institute of Pediatric Research, Children's Hospital of Hebei Province, 133 Jianhua South Street, Shijiazhuang 050031, Hebei Province, China. ${ }^{2}$ Hebei General Hospital, Shijiazhuang 050000, China. ${ }^{3}$ No.2 Department of Respiratory, Children's Hospital of Hebei Province, 133 Jianhua South Street, Shijiazhuang 050031, Hebei Province, China.

Received: 15 February 2019 Accepted: 4 November 2019

Published online: 12 November 2019

\section{References}

1. Tang VL, Halm EA, Fine MJ, Johnson CS, Anzueto A, Mortensen EM. Predictors of rehospitalization after admission for pneumonia in the veterans affairs healthcare system. J Hosp Med. 2014;9(6):379-83.

2. Toledo D, Soldevila N, Torner N, Perez-Lozano MJ, Espejo E, Navarro G, Egurrola M, Dominguez A. On-behalf of the project FISPIWG: factors associated with 30-day readmission after hospitalisation for communityacquired pneumonia in older patients: a cross-sectional study in seven Spanish regions. BMJ Open. 2018;8(3):e020243.

3. Torres A, Peetermans WE, Viegi G, Blasi F. Risk factors for communityacquired pneumonia in adults in Europe: a literature review. Thorax. 2013; 68(11):1057-65.
4. Capelastegui A, Espana Yandiola PP, Quintana JM, Bilbao A, Diez R, Pascual S, Pulido E, Egurrola M. Predictors of short-term rehospitalization following discharge of patients hospitalized with community-acquired pneumonia. Chest. 2009:136(4):1079-85.

5. Dong T, Cursio JF, Qadir S, Lindenauer PK, Ruhnke GW. Discharge disposition as an independent predictor of readmission among patients hospitalised for community-acquired pneumonia. Int J Clin Pract. 2017;71(3-4):e12935.

6. Jasti $H$, Mortensen EM, Obrosky DS, Kapoor WN, Fine MJ. Causes and risk factors for rehospitalization of patients hospitalized with communityacquired pneumonia. Clin Infect Dis. 2008;46(4):550-6.

7. Adamuz J, Viasus D, Camprecios-Rodriguez P, Canavate-Jurado O, JimenezMartinez E, Isla P, Garcia-Vidal C, Carratala J. A prospective cohort study of healthcare visits and rehospitalizations after discharge of patients with community-acquired pneumonia. Respirology. 2011;16(7):1119-26.

8. Brogan TV, Hall M, Williams DJ, Neuman MI, Grijalva CG, Farris RW, Shah SS. Variability in processes of care and outcomes among children hospitalized with community-acquired pneumonia. Pediatr Infect Dis J. 2012;31(10):1036-41.

9. Neuman MI, Hall M, Gay JC, Blaschke AJ, Williams DJ, Parikh K, Hersh AL, Brogan TV, Gerber JS, Grijalva CG, et al. Readmissions among children previously hospitalized with pneumonia. Pediatrics. 2014;134(1):100-9.

10. Nakamura MM, Zaslavsky AM, Toomey SL, Petty CR, Bryant MC, Geanacopoulos AT, Jha AK, Schuster MA. Pediatric Readmissions After Hospitalizations for Lower Respiratory Infections. Pediatrics. 2017;140(2): e20160938.

11. Berry JG, Toomey SL, Zaslavsky AM, Jha AK, Nakamura MM, Klein DJ, Feng JY, Shulman S, Chiang WW, Kaplan W, et al. Pediatric readmission prevalence and variability across hospitals. Jama. 2013;309(4):372-80.

12. Lee KY. Pediatric respiratory infections by mycoplasma pneumoniae. Expert Rev Anti-Infect Ther. 2008;6(4):509-21.

13. Nelson S, Belknap SM, Carlson RW, Dale D, DeBoisblanc B, Farkas S, Fotheringham $\mathrm{N}$, Ho H, Marrie $\mathrm{T}$, Movahhed $\mathrm{H}$, et al. A randomized controlled trial of filgrastim as an adjunct to antibiotics for treatment of hospitalized patients with community-acquired pneumonia. CAP study group. J Infect Dis. 1998;178(4):1075-80.

14. Barker CE, Sillis M, Wreghitt TG. Evaluation of Serodia Myco II particle agglutination test for detecting mycoplasma pneumoniae antibody: comparison with mu-capture ELISA and indirect immunofluorescence. J Clin Pathol. 1990;43(2):163-5

15. Templeton KE, Scheltinga SA, Graffelman AW, Van Schie JM, Crielaard JW, Sillekens P, Van Den Broek PJ, Goossens H, Beersma MF, Claas EC. Comparison and evaluation of real-time $P C R$, real-time nucleic acid sequence-based amplification, conventional PCR, and serology for diagnosis of mycoplasma pneumoniae. J Clin Microbiol. 2003;41(9):4366-71.

16. Suzuki S, Yamazaki T, Narita M, Okazaki N, Suzuki I, Andoh T, Matsuoka M, Kenri T, Arakawa Y, Sasaki T. Clinical evaluation of macrolide-resistant mycoplasma pneumoniae. Antimicrob Agents Chemother. 2006;50(2):709-12.

17. Wang L, Feng Z, Zhao M, Yang S, Yan X, Guo W, Shi Z, Li G. A comparison study between GeXP-based multiplex-PCR and serology assay for mycoplasma pneumoniae detection in children with community acquired pneumonia. BMC Infect Dis. 2017;17(1):518.

18. Wang L, Zhao M, Shi Z, Feng Z, Guo W, Yang S, Liu L, Li G. A GeXP-based assay for simultaneous detection of multiple viruses in hospitalized children with community acquired pneumonia. PLoS One. 2016;11(9):e0162411.

19. Driscoll AJ, Karron RA, Morpeth SC, Bhat N, Levine OS, Baggett HC, Brooks WA, Feikin DR, Hammitt LL, Howie SRC, et al. Standardization of Laboratory Methods for the PERCH Study. Clin Infect Dis. 2017;64(suppl_3):S245-52.

20. Marks C, Loehrer S, McCarthy D. Hospital readmissions: measuring for improvement, accountability, and patients. Issue brief. 2013;24:1-8.

21. Andruska A, Micek ST, Shindo Y, Hampton N, Colona B, McCormick S, Kollef $\mathrm{MH}$. Pneumonia pathogen characterization is an independent determinant of hospital readmission. Chest. 2015;148(1):103-11.

22. Toomey SL, Peltz A, Loren S, Tracy M, Williams K, Pengeroth L, Ste Marie A, Onorato S, Schuster MA. Potentially Preventable 30-Day Hospital Readmissions at a Children's Hospital. Pediatrics. 2016:138(2):e20154182.

23. Waites KB, Talkington DF. Mycoplasma pneumoniae and its role as a human pathogen. Clin Microbiol Rev. 2004:17(4):697-728 table of contents.

24. Williams DJ, Hall M, Brogan TV, Farris RW, Myers AL, Newland JG, Shah SS. Influenza coinfection and outcomes in children with complicated pneumonia. Arch Pediatr Adolesc Med. 2011;165(6):506-12. 
25. Brogan TV, Hall M, Sills MR, Fieldston ES, Simon HK, Mundorff MB, Fagbuyi DB, Shah SS. Hospital readmissions among children with H1N1 influenza infection. Hosp Pediatr. 2014;4(6):348-58.

26. Halm EA, Fine MJ, Kapoor WN, Singer DE, Marrie TJ, Siu AL. Instability on hospital discharge and the risk of adverse outcomes in patients with pneumonia. Arch Intern Med. 2002;162(11):1278-84.

27. Auerbach AD, Kripalani S, Vasilevskis EE, Sehgal N, Lindenauer PK, Metlay JP, Fletcher G, Ruhnke GW, Flanders SA, Kim C, et al. Preventability and causes of readmissions in a National Cohort of general medicine patients. JAMA Intern Med. 2016;176(4):484-93.

28. Gay JC, Hain PD, Grantham JA, Saville BR. Epidemiology of 15-day readmissions to a Children's hospital. Pediatrics. 2011;127(6):e1505-12.

\section{Publisher's Note}

Springer Nature remains neutral with regard to jurisdictional claims in published maps and institutional affiliations.

Ready to submit your research? Choose BMC and benefit from:

- fast, convenient online submission

- thorough peer review by experienced researchers in your field

- rapid publication on acceptance

- support for research data, including large and complex data types

- gold Open Access which fosters wider collaboration and increased citations

- maximum visibility for your research: over $100 \mathrm{M}$ website views per year

At BMC, research is always in progress.

Learn more biomedcentral.com/submissions 